\title{
On the genetic diversity of spiny mice (genus Acomys) and gerbils (genus Gerbillus) in the Arabian Peninsula
}

\author{
Timothy C. Bray ${ }^{1,2 *}$, Nigel C. Bennett ${ }^{2}$, Osama B. Mohammed ${ }^{1}$ \\ and Abdulaziz N. Alagaili ${ }^{1}$ \\ ${ }^{I}$ Department of Zoology, King Saud University, Riyadh, Saudi Arabia; \\ ${ }^{2}$ Department of Zoology and Entomology, University of Pretoria, South Africa
}

\begin{abstract}
Using non-destructive sampling we provide further genetic characterisations for spiny mice (Acomys dimidiatus/cahirinus) and gerbils (Gerbillus sp.) in three regions in Saudi Arabia. All individuals were sequenced for a fragment of the cytochrome $b$ gene, and compared against available conspecifics and closely related taxa. We confirm the existence of a second Acomys dimidiatus/cahirinus lineage specific to the Arabian Peninsula as seen previously. The Arabian Gerbillus nanus is shown to group with Middle Eastern rather than African conspecifics. A second cryptic Gerbillus lineage was also sampled across multiple locations, which may be an uncharacterised G. dasyurus.
\end{abstract}

Keywords: Acomys; genetic diversity; Gerbillus; rodents; Saudi Arabia.

\section{Introduction}

Partially due to its diversity of continental connections, the fauna of the Arabian Peninsula reflects a mixture of influences, the majority of studies describing Afro-Arabian affinities across different taxonomic groups (e.g. Pook, Joger, Stumpel, \& Wuster, 2009). In line with predictions of high rodent diversity in arid regions (Abramsky \& Rosenzweig, 1984), Arabian rodents include species of Acomys, Eliomys, Gerbillus, Jaculus, Meriones, and Psammomys. Among these taxa, the gerbil and spiny mouse are commonly found throughout the Arabian Peninsula, representing genera of Afro-Indian distribution. Studies on spiny mouse (Acomys) have included limited sampling in Arabia (Frynta et al., 2010), and some species of gerbil (Gerbillus) are as yet unrepresented in GenBank for gene regions of phylogenetic comparison (e.g. Gerbillus dasyurus) despite extensive recent work on African representatives (Ndiaye, Shanas, \& Granjon, 2013). Here we sample Acomys and Gerbillus across three Saudi Arabian locations each to give a preliminary indication of their geographical breaks and diversity in the Arabian Peninsula. Specifically we ask if there is a common trend in distribution of genetic variation across the taxonomic groups investigated, and how this relates to the same species in neighbouring regions.

\section{Material and Methods}

Gerbils $(n=17)$ and Spiny Mice $(n=16)$ were trapped and non-destructively sampled from three regions in the Kingdom of Saudi Arabia in March-June 2012. Locations sampled for both groups included Abha, Raydah Protected Area, and Taif areas with additional Acomys from Riyadh region and gerbils from Hawtat Bani Tamim (coordinates in Appendix 1).

DNA extraction was performed from earlobe tissue using Qiagen extraction reagents and separate spin columns (Epoch Life Science). The mitochondrial cytochrome $b$ (cytb) gene was amplified using the primers ALLROD/H15149 (Irwin, Kocher, \& Wilson, 1991) for gerbils, and for the spiny mice MOLCIT/MVZ (Ibáňez, García-Mudarra, Ruedi, Stadelmann, \& Juste, 2006; Smith \&

${ }^{*}$ Corresponding author. Email: tim_c_bray@hotmail.com 
Patton, 1993). A shorter spiny mouse fragment was amplified with MTCBF/H15149 (Naidu, Fitak, Munguia-Vega, Culver, 2012; Irwin et al., 1991; all cytb was amplified at $50^{\circ} \mathrm{C}$ for 40 cycles). A subgroup of gerbils was additionally sequenced for a fragment of the cytochrome oxidase c subunit I (COI) using universal primers LCOI490/HCO2198 (Folmer, Black, Hoeh, Lutz, \& Vrijenhoek, 1994), and a fragment of the 16s ribosomal gene (16s) using universal primers COIF/COIR (Palumbi \& Benzie, 1991; 40 cycles at $55^{\circ} \mathrm{C}$ ). Denaturation and extension temperatures for all primer combinations were 95 and $72^{\circ} \mathrm{C}$ respectively. All sequencing was performed on an ABI 3130 sequencing platform.

Sequence data was cleaned and aligned in BIOEDIT (Hall, 2005) and combined with samples available on GenBank (accession numbers in Appendix 1). Nucleotide substitutions and translation into amino acids was performed through MEGA5 (Tamura et al., 2011). Maximum parsimony haplotype networks were calculated using the TCS (Clement, Posada, \& Crandall, 2000) application. Model determination and construction of neighbour joining trees were conducted between gene regions separately (1000 bootstraps) using genetic distances inferred under the Tamura and Nei (1993) model for nucleotide sequences, in MEGA5. In this approach a maximum likelihood approach was used to produce phylogenetic trees showing support values for nodes with $>80 \%$ bootstrap support. Where two genes were available MRBAYES 3.1 (Ronquist \&

Huelsenbeck, 2003) was used. We used the default models for our gene partitions, setting MrBayes to infer coding bias assuming that only variable characters can be observed for both (“CODING = VARIABLE"). Bayesian analyses were undertaken using four independent runs, each using a random starting tree and $1 \times 10^{6}$ generations with one cold and three heated chains, sampling trees every 100 generations. The trees are derived from a consensus of the last 9901 trees for the first of the four independent runs (removing the initial 100).

\section{Results}

\section{Spiny Mouse Acomys sp.}

A total of 800 base pairs (bp) of cytb sequence data were generated across 16 spiny mice, with a further 29 sequenced for a shorter 404bp fragment. Of these longer 16 sequences; 10 were unique with sequence variation comprising 36 variable bases, 22 of these being parsimony informative. The $\mathrm{GTR}+\mathrm{G}$ model of sequence evolution was determined to be the best fit to the data $(\mathrm{AIC}=4845)$. Initially a neighbor joining phylogeny was constructed for the greater $A$. cahirinus/dimidiatus species group, but this was reduced to exclude the ' $A$. minous branch' to concentrate on the sampled lineages (Figure 1a). A haplotype network with this reduced $A$. cahirinus/dimidiatus set from GenBank identified 19 haplotypes and a split with a minimum distance of 16 substitutions between the southern Saudi Arabian samples and the next closest haplotype (Figure $1 b)$.

\section{Gerbils Gerbillus sp.}

For cytb 404bp were generated for 18 individuals, giving 13 unique sequences. Sequence variation comprised 70 variable bases, 53 of these being parsimony informative. The $\mathrm{GTR}+\mathrm{G}$ model of sequence evolution was determined to be the best fit to the data $(\mathrm{AIC}=3513)$. Both the neighbor joining phylogeny (Figure $2 \mathrm{a})$, and haplotype network (Figure 2b) showed that of the sampled Gerbillus, nine individuals fell within the GenBank representatives of $G$. nanus, and a further eight individuals (of mixed localities) fell outside this group.

To further explore the discrepancy between the deeper split within the Saudi Arabian Gerbillus samples two additional genes were sequenced across this split; seven unique $656 \mathrm{bp}$ COI fragments $(\mathrm{n}=7)$ and 3 unique $570 \mathrm{bp} 16$ s sequences $(\mathrm{n}=7)$ were re- 
covered. A three-gene combined MRBAYES consensus tree provided a well supported divergence between these lineages (Appendix 3).

\section{Discussion}

Tilman's prediction of high desert diversity has been shown to hold true at least in rodents (Abramsky \& Rosenzweig, 1984), suggesting that arid environments are particularly important for small mammal species assemblages. Most small mammal species in Saudi Arabia are genetically represented by only a small number of individuals from a single location. By sampling three small mammals from multiple locations we greatly increase the available knowledge of these species in this region, as can be seen with just a cursory glance at the figures presented here.

Within G. nanus there appears to be a break at the western edge of the Arabian Peninsula as shown in Ndiaye et al. (2013), with Libyan specimens grouping alongside those further west. The Saudi Arabian G. nanus groups more closely with haplotypes from Israel as well as the haplotype available from Pakistan, in line with this previously recognised split. Of interest here is that of the Saudi Arabian Gerbillus sampled was a lineage clearly divergent from the other G. nanus. This clade was present in both the southern and western sampling locations (although there is divergence between locations). What is currently unclear is whether this divergent lineage represents the currently genetically uncharacterised $G$. dasyurus. Further combined morphological and genetic determination will be necessary to clarify this issue. If there is regional ecological differentiation, as suggested for the spiny mouse, the absence of the recognised $G$. nanus in the south might represent its replacement in this area. More exhaustive sampling is necessary to better confirm species ranges in these species.

\section{Acknowledgements}

This project was supported by King Saud University, Deanship of Scientific Research, College of Science Research Centre, in collaboration with the DST-NRF SARChI Chair for Mammal behavioural ecology and physiology. We would like to acknowledge HH Prince Bandar Bin Mohammed Bin Saud, President of Saudi Wildlife Authority, for permissions to trap animals in the Raydah Protected Area. Thanks are also due to J. Sarli, T. Wronski, George, and others for assistance with the sampling effort, as well as an anonymous reviewer and the journal editor for invaluable advice on improving this manuscript.

\section{References}

Abramsky, Z., \& Rosenzweig, M. L. (1984): Tilman's predicted productivity-diversity relationship shown by desert rodents. Nature, 309, 150-151.

Clement, M., Posada, D., \& Crandall, K. A. (2000). TCS: a computer program to estimate gene genealogies. Molecular Ecology, 9, 1657-1660.

Fernandes, C. A. (2011). Colonization time of Arabia by the White-tailed Mongoose Ichneumia albicauda as inferred from mitochondrial DNA sequences. Biodiversity Conservation in the Arabian Peninsula Zoology in the Middle East, Supplementum, 3, 111-124.

Folmer, O., Black, M., Hoeh, W., Lutz, R., Vrijenhoek, R. (1994): DNA primers for amplification of mitochondrial cytochrome c oxidase subunit I from diverse metazoan invertebrates. Molecular Marine Biology and Biotechnology, 5, 294-299.

Frynta, D., Palupcikova, K., Bellinvia, E., Benda, P., Skarlantova, H., Schwarzova, L., \& Modry, D. (2010): Phylogenetic relationships within the cahirinus-dimidiatus group of the genus Acomys (Rodentia: Muridae): new mitochondrial lineages from Sahara, Iran and the Arabian Peninsula. Zootaxa, 2660, 46-56. 
Hall, T. (2005): BioEdit v 7.0.5. http://www.mbio.ncsu.edu/Bio-Edit.

Ibáňez C., García-Mudarra, J. L., Ruedi, M., Stadelmann, B., \& Juste, J. (2006): The Iberian contribution to cryptic diversity in European bats. Acta Chiropterologica, 8, 277-297.

Irwin, D. M., Kocher, T. D., \& Wilson, A. C. (1991): Evolution of the cytochrome b gene of mammals. Journal of Molecular Evolution, 32, 128-144.

Naidu, A., Fitak, R. R., Munguia-Vega, A., \& Culver, M. (2012): Novel primers for complete mitochondrial cytochrome b gene sequencing in mammals. Molecular Ecology Resources, 12, 191-196

Ndiaye, A., Shanas, P., \& Granjon, L. (2013): Molecular variation and chromosomal stability within Gerbillus nanus (Rodentia, Gerbillinae): taxonomic and biogeographic implications. Mammalia, 77, 105-111.

Palumbi, S. R., \& Benzie, J. (1991): Large mitochondrial DNA differences between morphologically similar penaeid shrimp. Molecular marine Biology and Biotechnology, 1, 27-34

Pook, C. E., Joger, U., Stumpel, N., \& Wuster, W. (2009). When continents collide: phylogeny, historical biogeography and systematics of the medically important viper genus Echis (Squamata: Serpentes: Viperidae). Molecular Phylogenetics and Evolution, 53, 792-807.

Racey, P. A., Barratt, E. M., Burland, T. M., Deaville, R., Gotelli, D., et al. (2007): Microsatellite DNA polymorphism confirms reproductive isolation and reveals differences in population genetic structure of cryptic pipistrelle bat species. Biological Journal of the Linnean Society, 90, 539-550.

Ronquist, F., \& Huelsenbeck, J. P. (2003): MrBayes 3: Bayesian phylogenetic inference under mixed models. Bioinformatics, 19, 1572-1574.

Smith, M. F., \& Patton, J. L. (1993). The diversification of South American murid rodents: evidence from mitochondrial DNA sequence data for acodontine tribe. Biological Journal of the Linnean Society, 50, 149-177.

Tamura, K., Peterson, D., Peterson, N., Stecher, G., Nei, M., \& Kumar, S. (2011): MEGA5: Molecular evolutionary genetics analysis using maximum likelihood, evolutionary distance, and maximum parsimony methods. Molecular Biology and Evolution, 28, 2731-2739.

Tamura, K., \& Nei, M. (1993): Estimation of the number of nucleotide substitutions in the control region of mitochondrial DNA in humans and chimpanzees. Molecular Biology and Evolution, $10,512-526$.

Volobouev, V., Auffray, J. C., Debat, V., Denys, C., Gautun, J. C., \& Tranier, M. (2007): Species delimitation in the Acomys cahirinus-dimidiatus complex (Rodentia, Muridae) inferred from chromosomal and morphological analyses. Biological Journal of the Linnean Society, 91, 203-214.

\section{Appendix}

Appendix 1. Accession numbers for all sequence data collected during this study.

Appendix 2. References for additional samples used as described in appendix 2.

Appendix 3. A combined consensus Bayesian phylogeny for Gerbillus in Saudi Arabia using cytb, COI, and 16s showing support values for each node. 


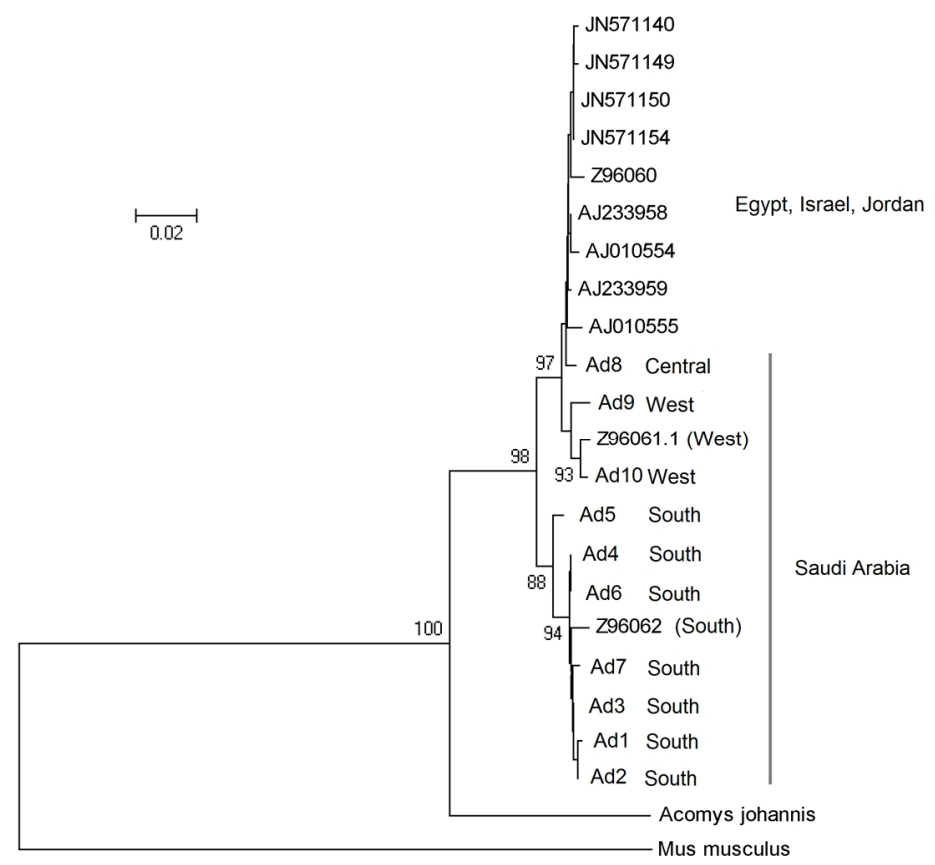

Figure 1a. A cytochrome b neighbour joining phylogeny for Acomys cahirinus/dimidiatus (a $\mathrm{GTR}+\mathrm{G}$ model of sequence evolution was used, bootstrap support values $>80 \%$ presented).

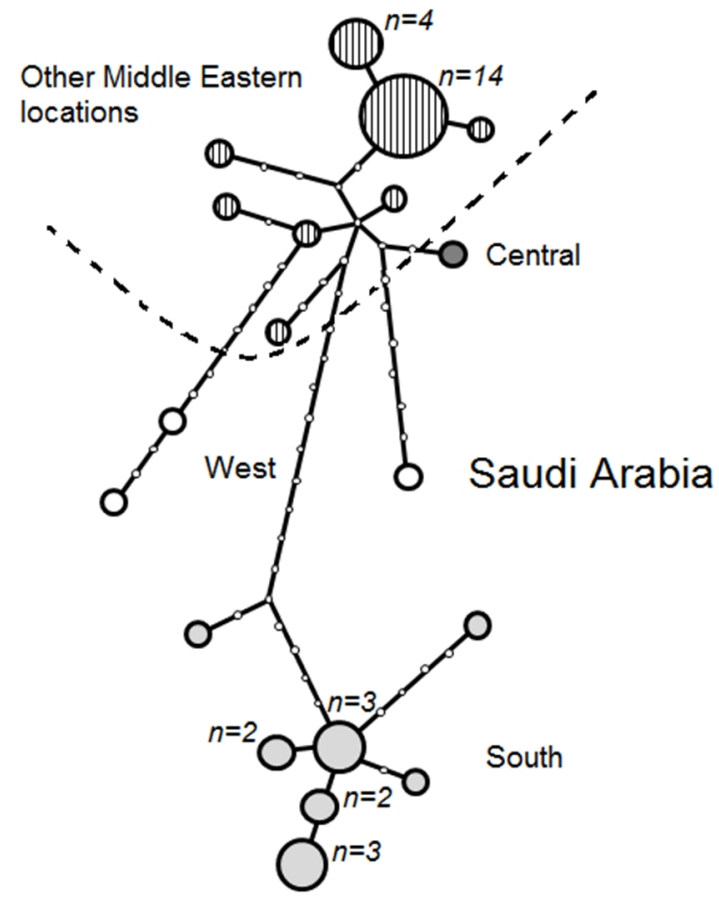

Figure 1b. Maximum parsimony haplotype network for Acomys dimidiatus showing numbers of individuals for each haplotype and numbers of mutational steps between them (small circles represent single steps). 


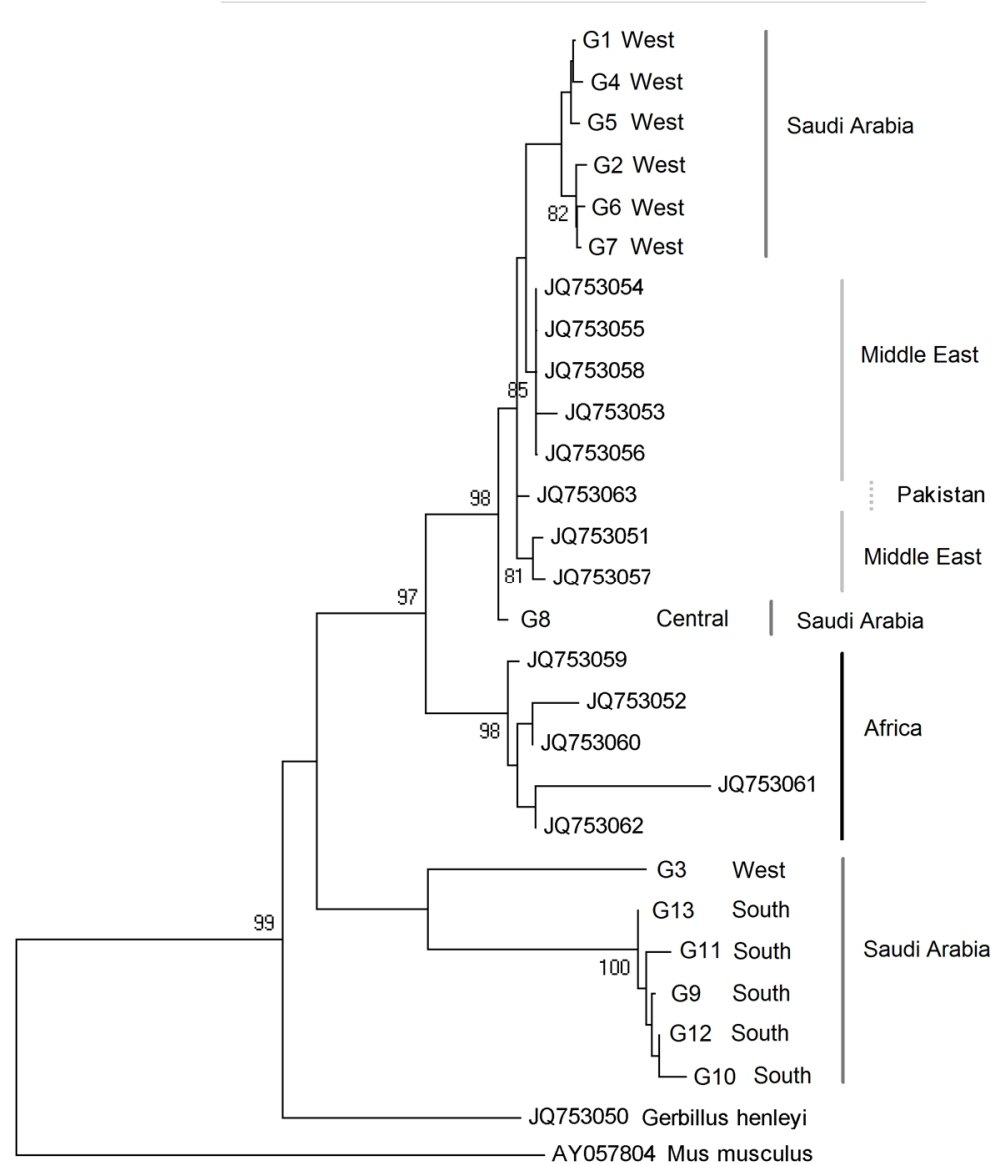

Figure 2a. A cytochrome $\mathrm{b}$ neighbor joining phylogeny for Gerbillus nanus (GTR $+\mathrm{G}$ model of sequence evolution used, bootstrap support values $>80 \%$ presented). 


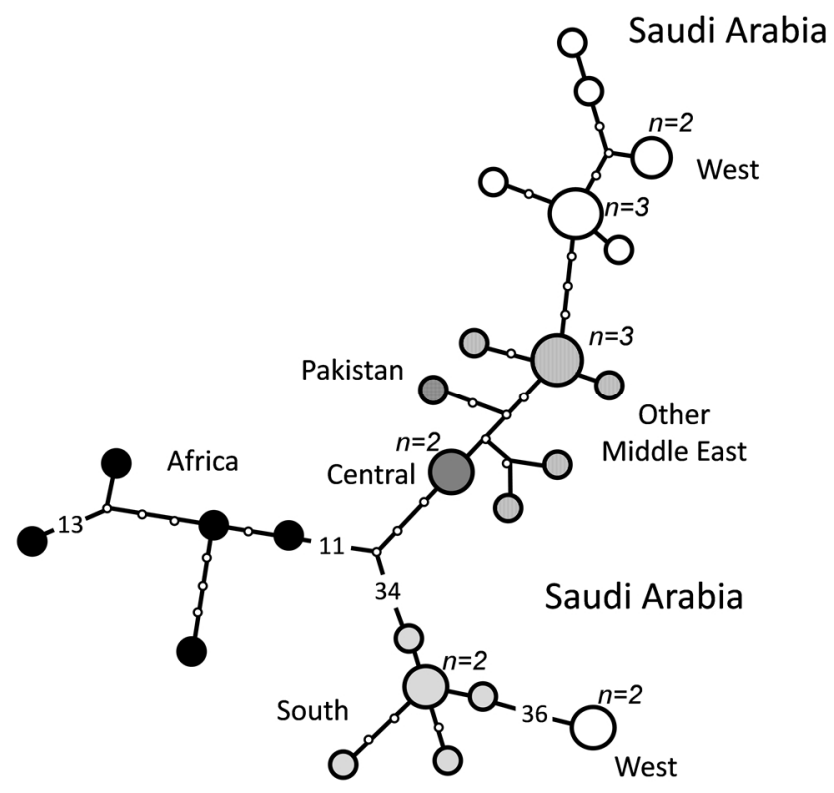

Figure 2b. Maximum parsimony haplotype network for Gerbillus nanus from Saudi Arabia showing numbers of individuals for each haplotype and numbers of mutational steps between them (small circles represent single steps). 


\section{Appendix 1}

\section{GENBANK accession numbers and sequence details (additional references in Appendix 2)}

\section{Cytochrome b gene}

\begin{tabular}{|c|c|c|c|c|}
\hline Species & $\mathbf{N}$ & Origin & GenBank & Reference \\
\hline Acomys dimidiatus & 3 & $\begin{array}{l}\text { South. Ad1 Raydah, Saudi Arabia } \\
18^{\circ} 02^{\prime} \mathrm{N}, 42^{\circ} 50^{\prime} \mathrm{E}\end{array}$ & KF422682 & This study \\
\hline A. dimidiatus & 2 & $\begin{array}{l}\text { South. Ad2 Raydah, Saudi Arabia } \\
18^{\circ} 02^{\prime} \mathrm{N}, 42^{\circ} 50^{\prime} \mathrm{E}\end{array}$ & KF422683 & This study \\
\hline A. dimidiatus & 3 & $\begin{array}{l}\text { South. Ad3 Tamniah, Saudi Arabia } \\
18^{\circ} 01^{\prime} \mathrm{N}, 42^{\circ} 46^{\prime} \mathrm{E}\end{array}$ & KF422684 & This study \\
\hline A. dimidiatus & 1 & $\begin{array}{l}\text { South. Ad4 Raydah, Saudi Arabia } \\
18^{\circ} 02^{\prime} \mathrm{N}, 42^{\circ} 50^{\prime} \mathrm{E}\end{array}$ & KF422685 & This study \\
\hline A. dimidiatus & 1 & $\begin{array}{l}\text { South. Ad5 Raydah, Saudi Arabia } \\
18^{\circ} 02^{\prime} \mathrm{N}, 42^{\circ} 50^{\prime} \mathrm{E}\end{array}$ & KF422686 & This study \\
\hline A. dimidiatus & 1 & $\begin{array}{l}\text { South. Ad6 Raydah, Saudi Arabia } \\
18^{\circ} 02^{\prime} \mathrm{N}, 42^{\circ} 50^{\prime} \mathrm{E}\end{array}$ & KF422687 & This study \\
\hline A. dimidiatus & 1 & $\begin{array}{l}\text { South. Ad7 Abha, Saudi Arabia } \\
18^{\circ} 02^{\prime} \mathrm{N}, 42^{\circ} 50^{\prime} \mathrm{E}\end{array}$ & KF422688 & This study \\
\hline A. dimidiatus & 1 & $\begin{array}{l}\text { Central. Ad8 Thumama, Saudi Arabia } \\
25^{\circ} 13^{\prime} \mathrm{N}, 46^{\circ} 38^{\prime} \mathrm{E}\end{array}$ & KF422689 & This study \\
\hline A. dimidiatus & 2 & $\begin{array}{l}\text { West. Ad9 Taif, Saudi Arabia } \\
21^{\circ} 16^{\prime} \mathrm{N}, 40^{\circ} 41^{\prime} \mathrm{E}\end{array}$ & KF422690 & This study \\
\hline A. dimidiatus & 1 & $\begin{array}{l}\text { West. Ad } 10 \text { Taif, Saudi Arabia } \\
21^{\circ} 16^{\prime} \mathrm{N}, 40^{\circ} 41^{\prime} \mathrm{E}\end{array}$ & KF422691 & This study \\
\hline A. dimidiatus & & $\begin{array}{l}\text { West. Taif, Saudi Arabia } \\
21^{\circ} 16^{\prime} \mathrm{N}, 40^{\circ} 41^{\prime} \mathrm{E}\end{array}$ & Z96061 & Barome et al. 1998 \\
\hline A. johannis & & Cameroon & HM635822 & Dobigny et al. 2011 \\
\hline A. dimidiatus & 4 & (A101) Israel $35^{\circ} 03^{\prime} \mathrm{N}, 3243.8^{\prime} \mathrm{E}$ & JN571140 & Hadid unpublished \\
\hline A. dimidiatus & 13 & (E22) Israel $35^{\circ} 03^{\prime} \mathrm{N}, 3243.8^{\prime} \mathrm{E}$ & JN571150 & Hadid unpublished \\
\hline
\end{tabular}




\begin{tabular}{|c|c|c|c|c|}
\hline Gerbillus nanus & 3 & $\begin{array}{l}\text { West. G1 Taif, Saudi Arabia } \\
21^{\circ} 16^{\prime} \mathrm{N}, 40^{\circ} 41^{\prime} \mathrm{E}\end{array}$ & KF422692 & This study \\
\hline Gerbillus nanus. & 2 & $\begin{array}{l}\text { West. G2 Taif, Saudi Arabia } \\
21^{\circ} 16^{\prime} \mathrm{N}, 40^{\circ} 41^{\prime} \mathrm{E}\end{array}$ & KF422693 & This study \\
\hline Gerbillus sp. & 2 & $\begin{array}{l}\text { West. G3 Taif, Saudi Arabia } \\
21^{\circ} 16^{\prime} \mathrm{N}, 40^{\circ} 41^{\prime} \mathrm{E}\end{array}$ & KF422694 & This study \\
\hline Gerbillus nanus & 2 & $\begin{array}{l}\text { West. G4 Taif, Saudi Arabia } \\
21^{\circ} 16^{\prime} \mathrm{N}, 40^{\circ} 41^{\prime} \mathrm{E}\end{array}$ & KF422695 & This study \\
\hline Gerbillus nanus & 1 & $\begin{array}{l}\text { West. G5 Taif, Saudi Arabia } \\
21^{\circ} 16^{\prime} \mathrm{N}, 40^{\circ} 41^{\prime} \mathrm{E}\end{array}$ & KF422696 & This study \\
\hline Gerbillus nanus & 1 & $\begin{array}{l}\text { West. G6 Taif, Saudi Arabia } \\
21^{\circ} 16^{\prime} \mathrm{N}, 40^{\circ} 41^{\prime} \mathrm{E}\end{array}$ & KF422697 & This study \\
\hline Gerbillus nanus & & $\begin{array}{l}\text { West. G7 Taif, Saudi Arabia } \\
21^{\circ} 16^{\prime} \mathrm{N}, 40^{\circ} 41^{\prime} \mathrm{E}\end{array}$ & KF422698 & This study \\
\hline Gerbillus nanus & & $\begin{array}{l}\text { Central. G8 Hawtat, Saudi Arabia } \\
23^{\circ} 17^{\prime} N, 46^{\circ} 32^{\prime} E\end{array}$ & KF422699 & This study \\
\hline Gerbillus sp. & & $\begin{array}{l}\text { South. G9 Raydah, Saudi Arabia } \\
18^{\circ} 02^{\prime} \mathrm{N}, 42^{\circ} 50^{\prime} \mathrm{E}\end{array}$ & KF422700 & This study \\
\hline Gerbillus sp. & & $\begin{array}{l}\text { South. G10 Habala, Saudi Arabia } \\
18^{\circ} 02^{\prime} N, 42^{\circ} 50^{\prime} E\end{array}$ & KF422701 & This study \\
\hline Gerbillus sp. & & $\begin{array}{l}\text { South. G11 Habala, Saudi Arabia } \\
18^{\circ} 02^{\prime} \mathrm{N}, 42^{\circ} 50^{\prime} \mathrm{E}\end{array}$ & KF422702 & This study \\
\hline Gerbillus sp. & & $\begin{array}{l}\text { South. G12 Abha, Saudi Arabia } \\
18^{\circ} 02^{\prime} N, 42^{\circ} 50^{\prime} \mathrm{E}\end{array}$ & KF422703 & This study \\
\hline Gerbillus sp. & & $\begin{array}{l}\text { South. G13 Tamniah, Saudi Arabia } \\
18^{\circ} 01^{\prime} N, 42^{\circ} 46^{\prime} \mathrm{E}\end{array}$ & KF422704 & This study \\
\hline Gerbillus henleyi & & Niger & JQ753050 & Ndiaye et al. 2012 \\
\hline Gerbillus nanus & 13 & Mali - Pakistan & JQ753051-63 & Ndiaye et al. 2012 \\
\hline Mus musculus & & $\begin{array}{l}\text { South. Raydah, Saudi Arabia } \\
18^{\circ} 02^{\prime} N, 42^{\circ} 50^{\prime} \mathrm{E}\end{array}$ & AY057804 & Lundrigan et al. 2002 \\
\hline Eliomys melanurus & 1 & & KF422705 & This study \\
\hline Mus sp & 1 & $\begin{array}{l}\text { South. Raydah, Saudi Arabia } \\
18^{\circ} 02^{\prime} \mathrm{N}, 42^{\circ} 50^{\prime} \mathrm{E}\end{array}$ & KF422706 & This study \\
\hline Mus sp & 1 & $\begin{array}{l}\text { South. Raydah, Saudi Arabia } \\
18^{\circ} 02^{\prime} N, 42^{\circ} 50^{\prime} \mathrm{E}\end{array}$ & KF422707 & This study \\
\hline
\end{tabular}




\section{Cytochrome Oxidase I (COI) gene}

\begin{tabular}{|c|c|c|c|c|}
\hline Species & $\mathbf{N}$ & Origin & GenBank & Reference \\
\hline Gerbillus nanus & 1 & $\begin{array}{l}\text { West. COI1 Taif, Saudi Arabia } \\
21^{\circ} 16^{\prime} \mathrm{N}, 40^{\circ} 41^{\prime} \mathrm{E}\end{array}$ & KF422708 & This study \\
\hline Gerbillus nanus & 1 & $\begin{array}{l}\text { West. COI } 2 \text { Taif, Saudi Arabia } \\
21^{\circ} 16^{\prime} \mathrm{N}, 40^{\circ} 41^{\prime} \mathrm{E}\end{array}$ & KF422709 & This study \\
\hline Gerbillus nanus & 1 & $\begin{array}{l}\text { West. COI3 Taif, Saudi Arabia } \\
21^{\circ} 16^{\prime} N, 40^{\circ} 41^{\prime} E\end{array}$ & KF422710 & This study \\
\hline Gerbillus sp. & 1 & $\begin{array}{l}\text { West. COI } 4 \text { Taif, Saudi Arabia } \\
21^{\circ} 16^{\prime} \mathrm{N}, 40^{\circ} 41^{\prime} \mathrm{E}\end{array}$ & KF422711 & This study \\
\hline Gerbillus sp. & 1 & $\begin{array}{l}\text { West. COI5 Taif, Saudi Arabia } \\
21^{\circ} 16^{\prime} \mathrm{N}, 40^{\circ} 41^{\prime} \mathrm{E}\end{array}$ & KF422712 & This study \\
\hline Gerbillus nanus & 1 & $\begin{array}{l}\text { West. COI6 Taif, Saudi Arabia } \\
21^{\circ} 16^{\prime} \mathrm{N}, 40^{\circ} 41^{\prime} \mathrm{E}\end{array}$ & KF422713 & This study \\
\hline Gerbillus nanus & 1 & $\begin{array}{l}\text { West. COI7 Taif, Saudi Arabia } \\
21^{\circ} 16^{\prime} \mathrm{N}, 40^{\circ} 41^{\prime} \mathrm{E}\end{array}$ & KF422714 & This study \\
\hline Meriones libycus & & $\begin{array}{l}\text { COIMl Thumama, Saudi Arabia } \\
25^{\circ} 13^{\prime} \mathrm{N}, 46^{\circ} 38^{\prime} \mathrm{E}\end{array}$ & KF422715 & This study \\
\hline Mus musculus & & - & AB444046 & $\begin{array}{l}\text { Nakamura et al } \\
\text { unpublished }\end{array}$ \\
\hline
\end{tabular}

\section{6s Ribosomal gene}

\begin{tabular}{|c|c|c|c|c|}
\hline Species & $\mathbf{N}$ & Origin & GenBank & Reference \\
\hline Gerbillus sp. & 2 & $\begin{array}{l}\text { West. G161 Taif, Saudi Arabia } \\
21^{\circ} 16^{\prime} \mathrm{N}, 40^{\circ} 41^{\prime} \mathrm{E}\end{array}$ & KF422716 & This study \\
\hline Gerbillus nanus & 3 & $\begin{array}{l}\text { West. G162 Taif, Saudi Arabia } \\
21^{\circ} 16^{\prime} \mathrm{N}, 40^{\circ} 41^{\prime} \mathrm{E}\end{array}$ & KF422717 & This study \\
\hline Gerbillus nanus & 1 & $\begin{array}{l}\text { West. G163 Taif, Saudi Arabia } \\
21^{\circ} 16^{\prime} \mathrm{N}, 40^{\circ} 41^{\prime} \mathrm{E}\end{array}$ & KF422718 & This study \\
\hline Meriones libycus & & $\begin{array}{l}\text { M11 Thumama, Saudi Arabia } \\
25^{\circ} 13^{\prime} \mathrm{N}, 46^{\circ} 38^{\prime} \mathrm{E}\end{array}$ & KF422719 & This study \\
\hline $\begin{array}{l}\text { Gerbillus nigeriae } \\
\text { Mus musculus }\end{array}$ & & $\begin{array}{l}\text { Mauritania } \\
\text { - }\end{array}$ & $\begin{array}{l}\text { AF141257 } \\
\text { AP013031 }\end{array}$ & $\begin{array}{l}\text { Ducroz et al. } 2001 \\
\text { Imanishi et al. } \\
\text { unpublished }\end{array}$ \\
\hline
\end{tabular}




\section{Appendix 2 \\ References additional to main text}

Barome, P. O., Monnerot, M., \& Gautun, J. C. (1998): Intrageneric phylogeny of Acomys (Rodentia, Muridae) using mitochondrial gene cytochrome b. Molecular Phylogenetics and Evolution 9, 560-566.

Dobigny, G., Tatard,C., Kane,M., Gauthier,P., Brouat,C., Ba,K. \& Duplantier,J.-M. A cytotaxonomic and DNA-based survey of rodents from Northern Cameroon and Western Chad. Mammalian Biology 76 (4), 417-427 (2011)

Ducroz,J.F., Volobouev,V. \& Granjon,L. (2001): An assessment of the systematics of arvicanthine rodents using mitochondrial DNA sequences: evolutionary and biogeographical implications. Journal of Mammalian Evolution 8 (3), 173-206

Hadid, Y. (Unpublished): Genetic divergence and incipient speciation in common spiny mice, Acomys cahirinus, at 'Evolution Canyon'.

Imanishi, H., Takibuchi, G. \& Hayashi, J. (Unpublished): Polymorphic mutations in mouse mitochondrial DNA regulate a tumor phenotype.

Lundrigan B.L., Jansa S.A., \& Tucker P.K. (2002): Phylogenetic relationships in the genus mus, based on paternally, maternally, and biparentally inherited characters. Systematic Biology 51(3), 410-431.

Nakamura,I., Ohnuma,A. \& Ichihashi,T. (Unpublished): Mus musculus mitochondrial COI gene for cytochrome c oxidase subunit I, partial cds.

Ndiaye A. Shanas U, Pascale C, \& Granjon L. (2012): Molecular variation and chromosomal stability within Gerbillus nanus (Rodentia, Gerbillinae): taxonomic and biogeographic implications. Mammalia 0, $1-7$. 
Appendix 3. A combined consensus Bayesian phylogeny for Gerbillus in Saudi Arabia using cytb, COI, and 16s showing support values for each node.

AY057804 Mus musculus

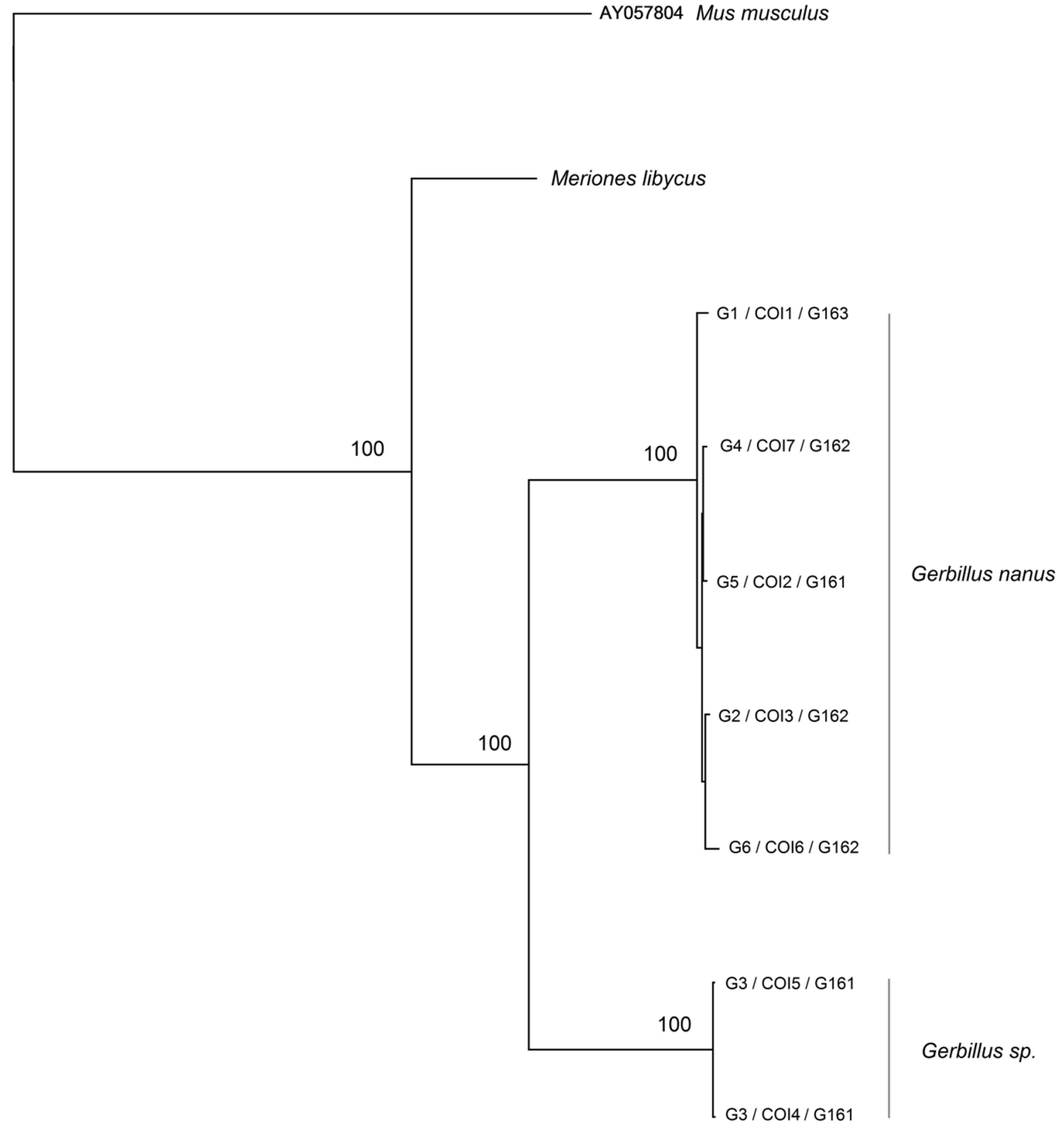

